\title{
Construire la confiance, une vertu nouvelle pour sortir du désir mimétique et de la violence
}

Gilles Le Cardinal, Jean-François Guyonnet et Bernard Pouzoullic

\section{(2) OpenEdition}

12 Journals

Édition électronique

URL : http://journals.openedition.org/communicationorganisation/2283

DOI : 10.4000/communicationorganisation.2283

ISSN : $1775-3546$

Éditeur

Presses universitaires de Bordeaux

Édition imprimée

Date de publication : 1 novembre 1999

ISSN : 1168-5549

\section{Référence électronique}

Gilles Le Cardinal, Jean-François Guyonnet et Bernard Pouzoullic, « Construire la confiance, une vertu nouvelle pour sortir du désir mimétique et de la violence », Communication et organisation [En ligne], 16 | 1999, mis en ligne le 19 décembre 2012, consulté le 30 avril 2019. URL : http:// journals.openedition.org/communicationorganisation/2283; DOI : 10.4000/ communicationorganisation.2283

Ce document a été généré automatiquement le 30 avril 2019

(C) Presses universitaires de Bordeaux 


\title{
Construire la confiance, une vertu nouvelle pour sortir du désir mimétique et de la violence
}

\author{
Gilles Le Cardinal, Jean-François Guyonnet et Bernard Pouzoullic
}

\section{Introduction}

1 Depuis une décennie, le concept de confiance sort peu à peu des préjugés multiples de naïveté, d'angélisme, de sentiment enfantin ou au contraire, de moyen de manipulation qui l'entouraient, pour gagner l'intérêt de chercheurs de disciplines de plus en plus variées.

2 En 1989, nous présentions une modélisation systémique de la communication interpersonnelle finalisée (L'homme communiqué comme unique, thèse d'habilitation à diriger des recherches, Bordeaux 3), qui essayait de décrire les processus de construction et de déconstruction de la confiance comme une face cachée de l'interaction. Les réactions à ces travaux, recueillies au colloque «Du Mépris à la Confiance » organisé en 1990 à l'Université de Technologie de Compiègne (UTC), s'étalent sur une longue palette de prises de position : depuis la réflexion « cet objet d'étude n'existe pas » en passant par des remarques désabusées « que peut-il sortir d'intéressant pour les sciences humaines de la théorie des jeux?", jusqu'à un soutien appuyé de quelques-unes qui nous ont encouragés en nous donnant les moyens contractuels de poursuivre notre recherche et d'aller jusqu'à les appliquer dans le domaine social (DIS de la Somme) et dans les entreprises (Renault, France-Télécom...).

3 Le défi fut relevé à travers une méthode destinée à favoriser la construction de la coopération dans les projets complexes («PAT-Miroir», marqué déposée en 1992) et un ouvrage exposant le cadre conceptuel et opérationnel que nous avions développé en lien avec un réseau de consultants et après avoir dépassé le cap des cent applications (La Dynamique de la Confiance, Dunod, 1997). 
Les économistes (colloque La confiance en question, Aix, 1996), les politiques (La société de confiance, Alain Peyrefitte, 1994), le management (Oser la confiance, B. Martin, V. Lenhardt, B. Jarrosson, 1996), les sociologues (Les conséquences de la modernité, A. Giddens, 1994), le Pape Jean-Paul II (Encyclique, Foi et raison, 1998), les psychologues et les philosophes (colloque Rationalité et confiance, Dijon, 1999) se sont intéressés au sujet, le reconnaissant cette fois comme un objet d'étude digne d'intérêt.

Cela ne veut pas dire que la confiance ait été abordée sans réticences ni mises en garde de toutes sortes. De nombreuses peurs se sont exprimées: instrumentalisation de la confiance, nouveau sésame du management, moyen de manipulation aux mains des sectes, ou enfin récupération pour un nouvel intégrisme moral ou religieux, voie ouverte à tous les fanatismes.

6 Nous voudrions poursuivre notre réflexion, cette fois sur les fondements axiologiques de la confiance en la reliant au paradigme du désir mimétique développé par René Girard et aux différentes stratégies dégagées en théorie des jeux autour du dilemme du prisonnier.

7 Pour cela, notre fil conducteur sera doublé: les conséquences psychologiques du caractère unique de chaque personne dès que se produit une rencontre et les difficultés intrinsèques que rencontre le désir d'identifier et de développer cet unique.

8 Dans cette perspective, la construction de la confiance apparaît être une vertu nouvelle que nous tenterons de situer par rapport aux vertus de justice et de courage.

\section{De l'unique à l'alter ego}

9 Si on nait unique, comment pourrait-on le savoir? Et comment peut-on le découvrir? Cet unique n'est d'ailleurs que potentiel car il ne s'est pas encore exprimé. Son expression n'est d'ailleurs en rien déterminée. Elle se développera dans l'interaction avec les proches et l'environnement.

10 C'est en effet, à travers l'interaction avec ce que l'enfant ne peut concevoir que comme "ses semblables", ses "alter ego », que vont se développer ses capacités cognitives et relationnelles. La fusion initiale et incontournable avec la mère va évoluer vers une interaction enfant-mère-monde, de plus en plus source de frustrations car de moins en moins comblante.

11 L'autre, qu'il soit parent ou frère, ne peut d'abord être perçu que comme un " alter ego ", un autre comme soi. Plus exactement, l'autre est ce qu'il est appelé à devenir, puisqu'il est plus développé que lui. L'autre est le modèle sur lequel, par mimétisme, il va conformer son « être au monde».

12 L'autre est l'image vivante de ce qu'il va devenir. L'enfant apprend d'abord en imitant l'autre, il apprend qu'il est capable d'apprendre, que les capacités d'apprentissage sont en lui. Ou s'arrêtera cet apprentissage fondé sur la reproduction d'un comportement modèle qui se révèle possible parce qu'on découvre en soi les potentiels nécessaires à l'imitation de la marche, de la parole, de l'écriture ?... Tout semble confirmer que l'autre est comme lui, qu'il est appelé à devenir comme l'autre. 


\section{De l'alter ego à l'alter}

13 Le premier « non » vient mettre un terme à cet univers mimétique pacifiant, rassurant de la simple reproduction. Une différence de volonté vient s'exprimer, mettant en évidence une différence de ressenti, d'émotion, d'évaluation, d'objectif, de représentation, ou peutêtre le refus d'une règle.

Une déchirure se produit dans la fusion, dans la dépendance, une expulsion de l'univers « du même » vers celui de la contre-dépendance.

15 C'est grâce a la confiance en l'autre d'abord absolue, puis à la confiance relative de cet autre en lui, grâce à un apprentissage de son humanité faite dans le cadre d'une relation pleine d'affection confirmant ses aptitudes, que cette confiance en soi peut grandir et lui permettre enfin la prise de risque du premier « non » à la dépendance.

C'est peut-être aussi un ressenti de ses propres limites, à travers un échec dans la possibilité de reproduire un comportement très précis dont il ne trouve pas le potentiel en lui, qui lui permet de découvrir "qu'il ne sera jamais comme l'autre». Cette découverte débouche, comme le fait remarquer S. Moscovici, sur le fait que l'autre "d'alter ego », d'autre soi-même, va devoir prendre le statut « d'alter » tout court, unique face à un unique.

17 Au-delà de cette qualité de l'autre qu'il n'a pas, qui devient différence entre eux révélatrice de leurs uniques, il va découvrir qu'il y a une relation que l'autre a et qu'il n'a pas, qu'il ne pourra jamais avoir de façon identique. Le tiers fait irruption dans son univers. La relation de la mère au père lui échappe, or elle a du prix aux yeux de la mère. Cette différence là est radicale. On pourrait voir ici une illustration du complexe d'ÆEdipe dans une interprétation qui aurait laissé de côté toute la composante sexuelle si essentielle pour Freud, pour ne retenir que sa figure relationnelle. L'enfant découvre peu à peu la place imprenable du père. Cette impossibilité de prendre la place du père est d'autant plus pénible, s'il s'y accroche avec l'énergie du désespoir.

18 Le combat de l'enfant, et en fait de chacun de nous, entre la sécurité apparente du "même ", de la simple reproduction de ce qui est déjà ou de ce qui a été, et le risque d'accueillir et de développer de l'unique, est décisif, plénier, total :

19 - soit il n'y a, comme semble le dire René Girard, que du désir mimétique, que du même à reproduire et donc à désirer et alors il n'y a rien d'autre à attendre,

- soit il y a de l'unique, de l'inouï, du nouveau, des différences et nous sommes lancés dans une aventure imprévisible, irréversible, pleine de promesses et de dangers. Alors un vrai désir entre dans l'univers du possible.

\section{Vers le vrai désir}

21 Osons affirmer ici qu'il y a place en chacun de nous pour un vrai désir, mais qu'il est par nature même, bien difficile à identifier car sans cesse menacé de son faux semblant, le désir mimétique. Il y a un vrai désir mais son objet n'est jamais clair, jamais simple car il nécessité d'aller puiser dans son potentiel spécifique pour l'actualiser, cette fois sans modèle, en se lançant dans l'inconnu en espérant rencontrer de nouveaux possibles, sans carte, en espérant que le sol ne lâchera pas sous nos pas d'explorateur. Oui, il y a un vrai 
désir qui consiste à explorer son unique, à développer ses talents, à faire donner son fruit à notre potentiel en actualisant notre unique. René Girard, en révélant l'universalité du désir mimétique et en semblant dénier ou oublier toute possibilité à l'existence d'un vrai désir, ne contribue-t-il pas lui aussi, après nous avoir si intelligemment éclairé sur l'importance capitale du désir mimétique, à notre enfermement en son sein, alors même qu'il veut nous mettre en garde contre ses effets maléfiques?

S'il y a place en une personne pour un vrai désir fondé sur un potentiel unique à actualiser, l'autre n'a plus alors à être un modèle et il peut être autre chose qu'un modèle. Le vrai désir de l'autre enfin n'a plus à être désiré car il ne correspond à aucun potentiel en soi. Le vrai désir doit être complètement inventé, exploré pas à pas sur un chemin qui ne se dévoile qu'en marchant qu'en prenant les risques de se tromper, de souffrir, d'interagir, de se confronter à la différence radicale des autres êtres. En nous retournant vers notre passé, n'est-il pas évident que les instants qui ont le plus de valeur à nos yeux sont ceux où notre unique s'est soudain révélé?

Alors se pose la question angoissante : comment découvrir son unique, son chemin, son vrai désir, ses potentiels spécifiques?

Il n'y a pas d'autre voie que l'interaction, la relation, le risque pris de coopérer avec l'autre. Cela suppose une confiance fondamentale dans le fait que nous partageons une commune humanité, que nous en sommes une incarnation unique, que les potentiels qui sont en nous ne pourront s'actualiser que si nos interactions permettent à ces potentiels uniques de s'exprimer, de se dévoiler mutuellement, de s'entre-révéler, de s'entreféconder.

25 Notons que l'interaction des libertés ne possède pas que Tissue favorable de cette coopération égalitaire où chacun partage ses informations, ses connaissances, son expérience pour une création commune. La trahison unilatérale et le conflit symétrique sont deux autres façons de vivre la relation à l'autre. La peur légitime de la souffrance qui en résulte vient obscurcir le vrai désir.

\section{Les stratégies possibles, d'après le dilemme du prisonnier}

26 La théorie des jeux a travers le dilemme du prisonnier, qui modélise une situation d'interaction où se pose le choix de la coopération ou de la non coopération, nous propose d'adopter l'une des stratégies suivantes:

27 - la stratégie prudente qui choisit d'éviter le pire ce qui empêche toute prise de risque, et donc toute coopération par nature risquée, et qui privilégie le rationnel pur,

28 - la stratégie dominante qui cherche à gagner plus, quel que soit le choix de l'autre et cède donc à toute opportunité de trahison,

29 - la stratégie donnant-donnant, dont le comportement mimétique reproduit le choix de l'autre à la séquence précédente en le laissant maitre du jeu et qui s'écroule dans les erreurs d'interprétation ou les aléas et qui suit les fous dans leur folie,

30 - la stratégie de Pavlov uniquement réactive, qui régule son action à l'aune de son revenu sans tenir compte de l'autre en rien (ni de son comportement ni de son revenu) et qui en se privant ainsi d'une partie de l'information, ne peut faire face à une situation complexe et incarne le déni de l'existence de l'autre. 

Confiance", ouvre de nouvelles perspectives, plus incertaines mais aussi pleines de promesses pour toutes les parties. Elle nous incite à tenir compte de tous les revenus, du sien et de celui de l'autre, à coupler les revenus des acteurs pour qu'en gagnant avec l'autre, on gagne plus que son revenu individuel, et qu'en perdant avec lui, la perte soit plus grande pour nous à cause de celle de l'autre.

$$
\text { la peur de la trahison et la tentation de trahir, tout en multipliant l'attrait à la }
$$
coopération.

34 À inverse, la méfiance contribue à transformer la situation en jeu en somme nulle où, on le sait, toute coopération apparait impossible.

Or, la coopération est seule vraiment féconde, puisque tout le monde gagne, la seule vraiment durable puisqu'elle accumule les gains avec le temps. La coopération mutuelle peut durer car elle crée des ressources, mais chacun sait qu'elle est hautement instable à moins qu'elle ne soit stabilisée par une confiance construite sur l'expérience passée à travers les promesses tenues, les fruits du projet commun partagés dans l'équité, les différences interpersonnelles reconnues et acceptées.

L'enjeu pour l'avenir est de faire émerger de petits noyaux humains unis par un projet, formant archipels, ouverts sur le monde, des «clusters" dit Axelrod en anglais, où s'installe la confiance, dont la taille et la maturité soient suffisantes pour faire la preuve de leur convivialité et de leur fécondité et donc de leur durabilité, même en milieu majoritairement non-coopératif.

\section{La différence, vraie source des peurs, attraits et tentations}

37 Notre hypothèse consiste ici à attribuer à la différence qui naît de la rencontre de plusieurs uniques partiellement encore inconnus, encore pleins de potentiels à naitre, les trois sentiments liés de peur, d'attrait et de tentation. Nous avions d'abord repéré ces trois sentiments dans le dilemme du prisonnier, archétype d'une interaction à fort enjeux. Nous en avions fait le fondement de la méthode PAT-Miroir (G. Le Cardinal, J.-F. Guyonnet, B. Pouzoullic), si utile en situations paradoxales. Cette nouvelle approche refonde les sentiments liés de peur, d'attrait et de tentation en les faisant exister dès la mise en présence de deux personnes avant même leur engagement dans une interaction forte.

38 Le vrai désir d'actualiser son potentiel unique est déjà un attrait puissant, même s'il ne s'actualisera qu'en l'engageant dans des interactions coopératives et fécondes avec l'unique de l'autre.

39 Le désir mimétique apparait alors à sa vraie place de «tentation du vrai désir » qui est refus d'accueillir les risques que comportent l'exploration de son unique au profit de ce que l'autre nous donne à voir. Cela n'enlève rien à son caractère universel mais ouvre la possibilité d'un autre désir.

Communication et organisation, $16 \mid 1999$ 
40 Si nous acceptons de reconnaitre et de prendre en compte la différence qui apparait dès le rapprochement de deux uniques, nous sommes alors livrés à la peur de l'inconnu en soi, en l'autre, avant même de subir les conséquences de leur interaction.

$$
\text { vaine }
$$
conduisent finalement à la violence ou à l'exclusion. Cette joie de la reconnaissance Alors nous pouvons dire, Avec Denis Vasse que la souffrance de la différence n'est acceptable que dans la joie de la reconnaissance des uniques. En effet l'homme tente mutuelle est rendue possible grâce à la prise des risques de l'interaction dans le respect Nous rajouterons que cette joie $\mathrm{n}$ 'est durable que grâce à la fécondité créatrice d'une coopération vécue dans la confiance.

53 À notre société qui voit se multiplier les trahisons au plan interpersonnel, social et politique en cette fin de $\mathrm{xx}^{\mathrm{e}}$ siècle et que l'on pourrait qualifier d'abandonnique suivant le concept inventé par M. Le May, il est utile d'ouvrir la possibilité, et si possible, de proposer le témoignage vivant de communautés humaines où la stratégie de la confiance produit son fruit durable, attirant, créatif et sans cesse renouvelé.

54 La confiance en l'autre permet, au nom de ce que l'on connaît de l'autre, d'accepter de s'engager avec lui malgré la part d'inconnu qui est en lui, sans recouvrir cet inconnu par ce que nous connaissons de nous-mêmes. 

permet seule la coopération créative de ces uniques. On sort ainsi de la simple reproduction de l'un sur l'autre, ou production d'un tiers identique à l'un ou à l'autre (clonage). On accède à la procréation, c'est-à-dire co-création du nouveau par l'alliance et l'interaction coopérative des uniques, dont le fruit «unique» contribue en retour à construire l'unique de ses procréateurs. yens, dans la souffrance de trahisons subies et dépassées, mais aussi dans l'espérance de la qualité de vie et de la fécondité qu'elle suscite au plus profond de nous, nous paraît être la vertu qu'appelle notre temps pour que le $\mathrm{xx}^{\mathrm{e}}$ siècle ne sombre pas dans le désir mimétique et la violence, dont René Girard a bien montré qu'ils étaient toujours associés. réflexions, pouvoir être une vertu nouvelle qui se situerait entre le courage (ou la force) et la justice.

69 
70 - La vertu de justice identifie les tentations qu'elle décrit dans la loi et sanctionne toutes trahisons de la peine juste.

71 Cette vertu de confiance serait à même de tempérer les peurs de l'altérité, de permettre de prendre les risques liés à l'interaction et de modérer les tentations du « même » après en avoir pris conscience. Donnant la main au courage et à la justice, encadrement qui lui permet de sortir définitivement de la naïveté première et de toutes les accusations d'enfantillage ou de manipulation, la vertu de la confiance rendrait possible la coopération durable en dépassant les peurs et les tentations qui y sont toujours associés.

72 Puissent les fruits de la dynamique de la confiance être assez désirables et sa gestion suffisamment attractive pour que nous puissions nous engager grâce à elle, dans les multiples prises de risques qu'implique la complexité du siècle à venir.

\section{BIBLIOGRAPHIE}

AXELROD R., Donnant-donnant, Odile Jacob, 1992.

BATESON G., Vers une écologie de l'esprit, Seuil, 1977, 1980.

BIBLE DE JERUSALEM, Cerf, 1973.

FREUD S., Nouvelles conférences d'introduction à la psychanalyse (trad. R. M. Zeitlin), 1933, Gallimard, N.R.F., Paris, 1984.

GIDDENS A, Les conséquences de la modernité, L’Harmattan, 1994.

GIRARD R., La violence et le sacré, Grasset, 1972.

GIRARD R., Les choses cachées depuis la création du monde, Grasset, 1978.

GIRARD R., Le bouc émissaire, Grasset, 1982.

GIRARD R., Quand ces choses commenceront, Arlea, 1994.

IDE P, Est-il possible de pardonner, Saint-Paul, 1994 ; Eh bien, dites don, 1997, Les éditions de l'Emmanuel.

JACQUARD A., Éloge de la différence, Seuil, 1978.

JACQUARD A., Cinq milliards d'hommes dans un vaisseau, Seuil, 1987.

LE CARDINAL G., GUYONNET J.-R, POUZoullic B., La dynamique de la confiance, Dunod, 1ère édition 1997, $2^{\mathrm{e}}$ impression 1999.

LE CARDinal G., GUYONnET J.-F, Du Mépris d la Confiance, actes du colloque UTC, 1990.

LE MAY M., J'ai mal à ma mère, Fleurus, 1979.

MARTIN B., LENHARDT V., JARROSSON B., Oser la confiance, INSEP, Édition 1996.

MOLINIER M.-D., Le courage d'avoir peur, Cerf, 1989.

Moscovici S., Psychologie sociale, PUF, 1984. 
NOWAK M., SIGMOND K., A strategy of win-stay, lose shift, that out performs, tit for tat with the Prisonner's dilemma game, Nature, vol. 364,1993.

PEYREFITTE A., La société de confiance, Odile Jacob, 1994.

RAPOPORT A., Prisonner Dilemma, Ann Arbor University, Michigan Press, 1965.

VASSE D., Le poids du réel, la souffrance, Seuil, 1983.

WinNicot D.W., Processus de maturation chez l'enfant, Payot, 1983.

WinNicot D.W., Jeu et réalité, l'espace potentiel, Gallimard, 1975.

\section{RÉSUMÉS}

Pouvons-nous sortir du désir mimétique et de la violence associée ? L'hypothèse consiste à poser l'existence d'un vrai désir, celui de découvrir son « unique », dont le désir mimétique ne serait que la tentation omniprésente. Le vrai désir pousserait à aller vers soi, dans l'ignorance de ce «soi ». L'unique de chaque personne ne se construit que dans l'interaction avec Tunique de l'autre. Les différences qui apparaissent alors peuvent devenir joie de la reconnaissance mutuelle, à condition de ne pas céder à la tentation du désir de l'unique de l'autre et de dépasser la souffrance inhérente aux différences. Ce chemin passe par la vertu d'une confiance bien gérée.

Can we escape mimetic desire and the violence associated with it? This hypothesis consists of assuming the existence of a true desire, that of discovering one's uniqueness, for which the mimetic desire would only be the omnipresent temptation. The true desire pushes us in search of oneself, in the ignorance of this "self ». The uniqueness of each person is only belt in the interaction with the uniqueness of another. The differences which appear can therefore become joys of mutual recognition, if we don't give in to the temptation of the desiring the uniqueness of the other and if we over-come the suffering caused by the differences. Such progress is only possible due to an acute awareness of the vitrue of carefully managed relationships of trust.

\section{INDEX}

Mots-clés : vrai désir, désir mimétique, confiance, souffrance, vertu

\section{AUTEURS}

\section{GILLES LE CARDINAL}

Gilles Le Cardinal, Professeur en Sciences de l'Information et de la Communication à Compiègne, est responsable de la formation doctorale et du DEA en Sciences Humaines et Technologie de la cognition et de la coopération. Son équipe a mis au point la méthode PAT-Miroir pour la (re)construction de la confiance et de la coopération dans les projets complexes (entreprises, établissements sociaux et scolaires). Il travaille depuis 1996 à la restauration de la qualité de la vie dans les territoires contaminés par la catastrophe de Tchernobyl. 